\title{
Masses of radiation pressure supported stars in extreme relativistic realm
}

\author{
Abhas Mitra \\ Theoretical Astrophysics Section, BARC, Mumbai-400085, India \\ email: abhasmitra@rediffmail.com
}

\begin{abstract}
We discuss that in the extreme relativistic limit, i.e., when $z \gg 1$, where $z$ is the surface gravitational redshift, there could be radiation pressure supported and dominated stars with arbitrary gravitational mass, high or low. Such objects are called Eternally Collapsing Objects (ECOs). ECOs are practically as compact as Schwarzschild black holes (BH) and, observationally, are likely to be mistaken as BHs. Further since any object undergoing continued collapse must first become an ECO before becoming a true BH state characterized by $M=0$, the observed BH Candidates are ECOs.
\end{abstract}

Keywords. Gravitation - black holes

\section{Introduction}

Let the ratio of radiation pressure to gas pressure in a star be $x=p_{r} / p_{g}$, and let the ratio of densities of radiation and rest masses be $y=\rho_{r} / \rho_{0}$. Then it follows that, within a factor of 2, $y \sim \alpha z$, where $\alpha=L / L_{e d}$ (Mitra 2006a). Here $L$ is the luminosity of the star and $L_{e d}$ is the maximal or Eddington luminosity of the same. It is also seen that $\alpha$ increase with $z$ and saturates to a maximal value of $\alpha \approx 1$. The conventional known "stars" have $z \ll 1$, i.e., they are Newtonian objects. In such a case $x \approx 0.2\left(M / M_{\odot}\right)^{1 / 2} \mu^{-1}$ where $\mu$ is the chemical composition and $M_{\odot}$ is solar mass (Mitra 2006b). In order to have a "radiation pressure supported star", one must have $x \gg 1$. Then the above equation demands that $M \gg M_{\odot}$. Such stars are known as (Newtonian) Supermassive Stars.

This picture changes dramatically when we allow for the likely occurrence of extreme relativistic situations with $z \gg 1$ when (Mitra 2006b)

$$
x \approx 0.25 z\left(\frac{M}{M_{\odot}}\right)^{1 / 2} \mu^{-1} .
$$

Because of the presence of $z$ on the RHS, now a radiation supported quasi-static state $(x \gg 1)$ can occur for arbitrary values of $M$. For instance, an object with $M=10^{10} M_{\odot}$ with a value of $z=100$ will have $x \gg 1$ and, on the other hand, a fireball of energy $1 \mathrm{TeV}$ created by two colliding elementary particles in a lab collider with $M=10^{-43} M_{\odot}$ could attain a radiation dominated quasi-static state with $z>10^{25}$ ! In both the cases, the radius of the object would be practically same as the radius of a supposed BH. Such a large value of $z$ may sound unphysical. However, it is not so because the continued collapse is supposed to proceed to the true $\mathrm{BH}$ state with $z=\infty$ (and all finite numbers including $10^{25}$ are of course infinitely smaller than $\infty$ ).

It has been shown that, as physical gravitational collapse must be radiative (Mitra 2006c) and $z$ keeps on increasing relentlessly during continued collapse, quanta of collapse generated photons and neutrinos must eventually move in almost closed circular orbits and be quasi-trapped even before an absolute capture would occur by the formation of trapped surfaces or Event Horizon (EH). It is this quasi-trapping of radiation which 
causes $x \gg 1$ and also $y \gg 1$. Since such states correspond to $\alpha \approx 1$, ECOs are formed much before a true BH state $(z=\infty)$ would be formed. Since the life time of this relativistic radiation dominated state is $t=5.10^{8}(1+z)$ yr and $t \rightarrow \infty$ as $z \rightarrow \infty$ ECOs are eternally collapsing (Mitra 2006d).

\section{Discussion}

As the ECOs evolve, their EOS $\rho \rightarrow 3 p$ and the gravitational mass $M=\int(\rho-3 p) d \Omega \rightarrow$ 0 where $d \Omega$ is element of proper volume (Mitra 2006e). Consequently, the eventual BH mass $M=0$ and thus the observed BHs with $M>0$, either discussed in this conferences or elsewhere, cannot be true BHs and must be ECOs. This conclusion that true BHs have $M \equiv 0$ has been also obtained by using the basic differential geometry property that proper spacetime 4-volume is coordinate independent (Mitra 2005, 2006f) and nonoccurrence of trapped surfaces and EH (Mitra 2002, 2004).

Since astrophysical plasma is endowed with intrinsic magnetic field the super compact ECOs must have strong intrinsic magnetic fields and hence they are also called Magnetospheric ECOs or MECOs. In contrast a true uncharged $\mathrm{BH}$ with an $\mathrm{EH}$ has no intrinsic magnetic field. Thus MECOs can be observationally distinguished from true BHs by using this physical property. And there are strong circumstantial evidences that the BH Candidates in (i) X-ray binaries (Robertson \& Leiter 2002, 2003, 2004), or the (ii) the compact object at the galactic centre (Robertson \& Leiter 2006) are MECOs with physical surfaces rather than true BHs. Most importantly, detailed microlensing aided mapping of the central engine of the one of the most well studied quasar has directly revealed that it is a MECO and not a BH (Schild, Leiter \& Robertson 2006). The observed magnetic field of MECOs will however be smaller by factor of $(1+z)$ and could be well below pulsar values. Because of joint effect of gravitational redshift and time dilatation, the observed luminosity of the ECOs would be also also extremely small, $L=1.3 \times 10^{38}\left(M / M_{\odot}\right)(1+z)^{-1} \mathrm{erg} / \mathrm{s}$ even though they are shining at their respective Eddington values. This feeble quiescent ECO radiation could be in millimeter, microwave or radio range and is yet to be detected for any individual case.

Since the ECO formation is a generic effect due to inevitable quasi-trapping of collapse generated radiation, continued collapse does not give rise to any mysterious phase transition resulting in negative pressure. Since Chandrasekhar mass limit pertains to cold objects, it is irrelevant for hot ECOs whose local mean temperature is reaches the value of $T=600\left(M / M_{\odot}\right)^{-1 / 2} \mathrm{MeV}$.

\section{References}

Mitra, A. 2006a, MNRAS, 367, L66, gr-qc/0601025

Mitra, A. 2006b, MNRAS, 369, 492, gr-qc/0603055

Mitra, A. 2006c, PRD, 74, 024010, gr-qc/0605066

Mitra, A. 2006d, New Astronomy, 12, 146, astro-ph/0608178

Mitra, A. 2006e, PRD, in press, gr-qc/0607087

Mitra, A. 2006f, Adv. Sp. Res., in press, astro-ph/0510162

Mitra, A. 2005, physics/0504076

Mitra, A. 2002, Found. Phys. Lett., 15, 439, astro-ph/0207056

Mitra, A. 2004, astro-ph/0408323

Robertson, S. L. \& Leiter, D. J. 2002, ApJ, 565, 447, astro-ph/0102381

Robertson, S. L. \& Leiter, D. J. 2003, ApJL, 596, L203, astro-ph/0310078

Robertson, S. L. \& Leiter, D. J. 2004, MNRAS, 350, 1391, astro-ph/0402445

Robertson, S. L. \& Leiter, D. J. 2006, astro-ph/0603746

Schild, R. E., Leiter, D. J. \& Robertson, S. L. 2006, AJ, 132, 420, astro-ph/0505518 